\title{
DcR3 regulates the growth and metastatic potential of SW480 colon cancer cells
}

\author{
WEI YU ${ }^{1 *}$, YING-CHEN XU $^{2 *}$, YING TAO $^{1}$, PING HE$^{2}$, YANG $^{2}{ }^{2}$, TAO WU $^{1}$, \\ YAN-PEI ZHU ${ }^{1}$, JING $\mathrm{LI}^{3}$, JI-XIANG WU ${ }^{4}$ and JIE DAI ${ }^{1}$ \\ ${ }^{1}$ Center for Clinical Pathology, Capital Medical University, Beijing 100069; ${ }^{2}$ Department of General Surgery, \\ Beijing Anzhen Hospital, Capital Medical University, Beijing 100029; ${ }^{3}$ Department of Pathology, \\ Beijing Aerospace General Hospital, Beijing 100076; ${ }^{4}$ Beijing Tongren Hospital, \\ Capital Medical University, Beijing 100730, P.R. China
}

Received August 5, 2013; Accepted September 2, 2013

DOI: $10.3892 /$ or.2013.2769

\begin{abstract}
Decoy receptor 3 (DcR3) is considered to have anti-apoptotic and pro-metastatic functions, suggesting it might be a therapeutic target. We examined the role and mechanisms of DcR3 on growth and the metastatic ability of SW480 colon cancer cells to provide therapeutic information for targeting DcR3 by RNA interference (RNAi) technology. Growth and the metastatic ability were inhibited, apoptosis was induced and cell cycle profile was changed after decreasing DcR3 expression, with lower levels of vascular endothelial growth factors (VEGFs) and matrix metalloproteinases (MMPs) expression. Our results implied the therapeutic potential of silencing DcR3 expression by RNAi in colon cancer.
\end{abstract}

\section{Introduction}

Decoy receptor 3 (DcR3), a soluble decoy receptor, is a member of the tumor necrosis factor receptor superfamily (1). DcR3 can prevent cell death and may play an important role in the pathogenesis of many malignancies by binding and inhibiting the function of the Fas receptor (2). Accumulating

Correspondence to: Professor Jie Dai, Center for Clinical Pathology, Capital Medical University, no. 10 Xitoutiao, You An Men, Fengtai, Beijing 100069, P.R. China

E-mail: sydaijie@126.com

Professor Ji-Xiang Wu, Beijing Tongren Hospital, Capital Medical University, no. 2 Chongwenmennei Street, Dongcheng, Beijing 100730, P.R. China

E-mail: trwujixiang@126.com

*Contributed equally

Abbreviations: DcR3, decoy receptor 3; RNAi, RNA interference; shRNA, short hairpin RNA; dsRNA, double-stranded RNA; VEGFs, vascular endothelial growth factors; MMPs, matrix metalloproteinases; GFP, green fluorescent protein; FCM, flow cytometry; PI, propidium iodide

Key words: DcR3, colon cancer, RNAi, MMPs, VEGFs evidence has demonstrated that DcR3 is overexpressed in several cancers $(3,4)$ and might protect cancer cells against the cytotoxic and regulatory effects of FasL, LIGHT19 and TNF-like molecule $(5,6)$. In addition, the expression of DcR3 positively correlated with tumor invasiveness in pancreatic cancer cells (7). DcR3 also has a described role as a tumor promoter in renal carcinoma, hepatoma, gastric carcinoma, and breast cancer $(6,8)$. DcR3 is expected to be an effective target for tumor gene therapy. However, little is known about the molecular mechanisms underlying the role of DcR3 in tumor progression and metastasis.

Colon cancer is one of the most common malignancies around the world, accounting for $8-15 \%$ of all cancer deaths (9). Colon cancer cells can co-opt signaling pathways stimulated by both exogenous factors and endogenous genetic agents for growth advantage and evasion of apoptosis (10). To explore the effects of DcR3 during tumorigenesis and progression of colon cancer, we determined the expression of DcR3 in a large number of colon cancer tissues. Cancerous colon tissues have high DcR3 expression and its expression is correlated with tumor differentiation, serosal invasion, Duck's stage and lymph node and liver metastases. Nevertheless, the precise mechanisms of DcR3 are not completely clear in progression and metastasis of colon cancer. Current therapies for colon cancer consist of excision, chemotherapy and radiotherapy, all of which have toxic or side-effects. Gene therapy has become a promising strategy for cancer treatment due to minimal drug resistance, high efficiency and specificity for drug delivery. In this study, we further determined the role of DcR3 in colon cancer, and assessed whether it may serve as a viable therapeutic target.

In recent years, RNA interference (RNAi) has shown great potential as a gene therapy strategy for cancer. Gene ablation technology involving short hairpin RNA (shRNA) synthesis can considerably suppress gene expression $(11,12)$. RNAi is mediated by small interfering RNAs (siRNAs) that are produced from double-stranded RNAs (dsRNAs) of exogenous or endogenous origin by an endonuclease of the ribonuclease-III type called Dicer. The resulting siRNAs are approximately 21-23 nucleotides (nt) long and are then incorporated into a nuclease complex, and transported to the 
targeted RNA for cleavage by the RNA-inducing silencing complex. shRNA transcribed from a vector are thought to suppress the expression of targeted genes more efficiently than synthesized siRNA $(13,14)$. In this study, we use a plasmid-based polymerase III promoter system for expressing shRNA to decrease DcR3 levels. We then assess its effects on the growth and invasive capability of SW480 colon cancer cells, to provide theoretical basis for DcR3 gene therapy by RNAi as a treatment strategy for colon cancer.

\section{Materials and methods}

Plasmid vector preparation. The plasmid vector (pGCSiU6-Neo-GFP-DcR3shRNA) carrying DcR3-shRNA was purchased from TransGen Biotech, Co., Ltd. (China). The sequence of DcR3-shRNA with a stem-loop structure: CGCT GGTTTCTGCTTGGAGCA- CTCGAG- TGCTCCAAGC AGAAACCAGCG. The plasmid vector of pGCSi-U6-NeoGFP-NegConshRNA (as the negative control) and the empty plasmid were purchased. To amplify the vectors, cloning was performed. The plasmid vectors were transformed into the competent $E$. coli $\mathrm{DH} 5 \alpha$ bacteria followed by ampicillin selection. The purified plasmid DNAs were tested for identification by digesting the clones with restriction endonuclease enzyme. The plasmids were extracted by Fastfiler Endo Free Plasmid Maxiprep kit (Omega Bio-Tek, USA).

Cell culture and experimental reagents. SW480 cells were cultured in Leibovitz's L-15 medium (Gibco, USA) supplemented with $10 \%$ fetal bovine serum (FBS), $100 \mathrm{U} / \mathrm{ml}$ penicillin and $100 \mu \mathrm{g} / \mathrm{ml}$ streptomycin, $100 \%$ atmospheric air without additional $\mathrm{CO}_{2}$ at $37^{\circ} \mathrm{C}$. The plasmid DNA was transfected into SW480 cells by Lipofectamine 2000 reagent (Invitrogen, USA), followed with G418 and flow cytometry (FACSVantage, BD Biosciences) for selecting stably transfected cells. Primary antibody of DcR3 (ab8405) was purchased from Abcam and other antibodies for vascular endothelial growth factor (VEGF)-C, VEGF-D, MT1-matrix metalloproteinase (MMP) and GAPDH were purchased from Santa Cruz Biotechnology, Inc. (Santa Cruz, CA, USA). Other reagents used in this study, such as anti-mouse-IgG-HRP and anti-rabbit-IgG-HRP, were purchased from California Biosciences (USA), Transwell Invasion Chambers were purchased from Corning (USA).

Plasmid transfection, stably transfected cell selection. Briefly, the day before transfection, $2 \times 10^{5}$ of cells were seeded in antibiotic-free L-15 medium into 6-well plates. The cells were transfected with a mixture of $3 \mu \mathrm{g}$ plasmids and $10.5 \mu \mathrm{l}$ of Lipofectamine 2000 reagent [ratio of plasmid DNA $(\mu \mathrm{g}) /$ Lipofectamine 2000 reagent $(\mu \mathrm{l})$ was $1: 3.5$, in our previous study] in $500 \mu \mathrm{l}$ medium per well. At $6 \mathrm{~h}$ post-transfection, the medium was replaced by complete medium, then cells were screened by $800 \mathrm{mg} / \mathrm{l} \mathrm{G} 418$ (the G418 concentration was adjusted according to cell viability, obtained from our previous study) and cultured up to 4 weeks. The DcR3-RNAi, negative control, and empty plasmid stable transfection cells were harvested. The cells were harvested by trypsinization and then resuspended in PBS at $4^{\circ} \mathrm{C}$. The mean fluorescent intensity of green fluorescent protein (GFP) from each group was analyzed by flow cytometry (FCM) (FACSAria; BD
Biosciences) through a $488 \mathrm{~nm}$ laser for excitation and the fluorescence emissions at $525 \mathrm{~nm}$ for GFP. The GFP positive cells were sorted. Stable cells of 3 groups were expanded. The expression levels of DcR3 in the 3 groups were examined by western blotting to detect the effect of DcR3 knockdown. Other experiments were performed as indicated.

Cell growth and viability studied by MTT assay. Cells of 3 groups were seeded with $100 \mu 1$ medium in a 96-well plate $\left(5 \times 10^{3}\right.$ per well), and the MTT assay was performed after incubation for indicated times. MTT reagent $(5 \mathrm{mg} / \mathrm{ml})$ was added to each well, and incubated for $4 \mathrm{~h}$ at $37^{\circ} \mathrm{C}$. The resulting formazan crystals were solubilized by the addition of $150 \mu \mathrm{l}$ DMSO to each well. The optical density at $490 \mathrm{~nm}$ was measured and cell viability was determined by the growth curve for 7 days. All MTT experiments were performed in triplicate and repeated at least three times.

Colony formation assay. DcR3 RNAi, empty plasmid, and control RNAi stable transfected cells were seeded in Leibovitz's L-15 medium supplemented with $10 \%$ FBS for 14 days prior to crystal violet staining for colony counting. Cells were then fixed in $4 \%$ paraformaldehyde and incubated in $0.01 \%$ crystal violet for $30 \mathrm{~min}$ for colony counting.

Monolayer cell migration assay. Cell migration was modeled using a wound-healing assay. DcR3 RNAi or empty plasmid/control RNAi stable transfected SW480 cells were seeded in 6-well plate for $24 \mathrm{~h}$ in L-15 medium and treated as above. The $100 \%$ confluent monolayer SW480 cells were then scraped with a sterile $100 \mu \mathrm{l}$ pipette tip and cell debris was washed with PBS. Cells that migrated into the wounded areas were photographed at the indicated times with an inverted microscope equipped with a digital camera. The extent of healing was defined as the ratio of the difference between the original and the remaining wound areas compared with the original wound area.

Transwell invasion assay. Matrigel Invasion Chambers were hydrated for $4 \mathrm{~h}$ prior to placing the cells into the chambers. Cells from each group were seeded at $1 \times 10^{5}$ cells in $200 \mu \mathrm{l}$ serum-free L-15 medium in the upper chambers of the Transwell and the lower chamber was filled with $500 \mu \mathrm{l}$ complete L-15 medium with $10 \%$ FBS. The cells were incubated for $48 \mathrm{~h}$ for the invasion assay. The cells remaining on the upper surface of the filter were removed carefully by cotton tips, and cells on the underside of the membrane were fixed with $4 \%$ paraformaldehyde. After washing the chambers 5 times by dipping the chambers in a large beaker filled with $\mathrm{dH}_{2} \mathrm{O}$, the cells remaining on the bottom of the chamber were stained with $0.1 \%$ crystal violet for $30 \mathrm{~min}$. The migrated cells were photographed under an optical microscope $(x 400)$ and counted at 12 different areas. The experiments were performed in triplicate and the data were compared with the controls.

Apoptosis and cell cycle analysis. DcR3 RNAi, control RNAi, and empty plasmid cells were cultured in the same conditions and harvested by trypsinization. The following steps were carried out in accordance with the apoptosis detection protocol. Cells were washed once in PBS, then once in $1 \mathrm{X}$ binding 


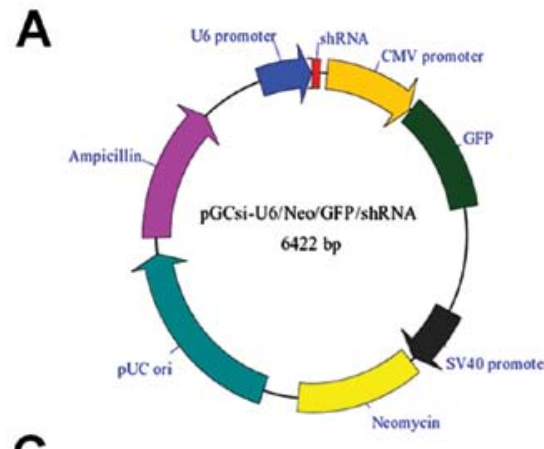

C

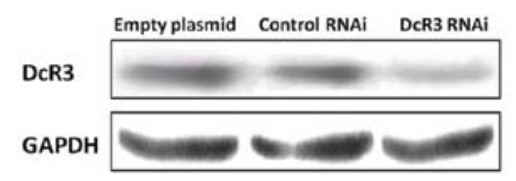

B
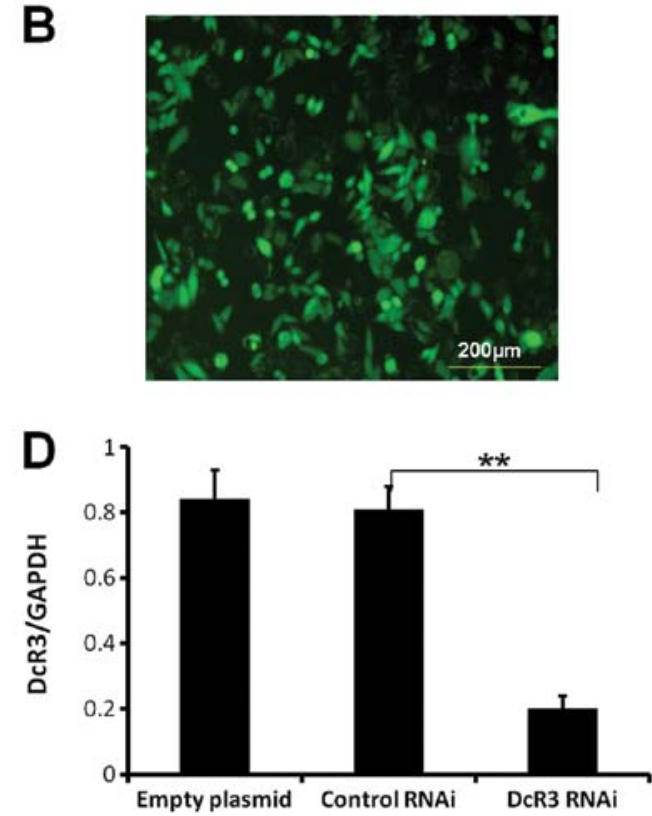

Figure 1. Structure of DcR3-shRNA plasmid and identification of stably transfected cells. (A) The features of DcR3-shRNA plasmid. (B) Stable expression of GFP in stably transfected cells with plasmid under inverted fluorescence. (C and D) Cells of all groups were harvested for DcR3 measurement by western blotting using GAPDH as an internal control. Expression of DcR3 was decreased significantly in DcR3 RNAi SW480 cells compared with controls. ${ }^{* *} \mathrm{P}<0.01$.

buffer, and resuspended in $1 \mathrm{X}$ binding buffer at $5 \times 10^{6} / \mathrm{ml}$. The cell suspension $(100 \mu \mathrm{l})$ was stained with $5 \mu \mathrm{l}$ of fluorochromeconjugated Annexin V, incubating $15 \mathrm{~min}$ at room temperature. Cells were washed and resuspended before adding $5 \mu \mathrm{l}$ of propidium iodide (PI). The samples were stored at $2-8^{\circ} \mathrm{C}$ in the dark before analyzing by FCM (EPICS XL Coulter Co., USA) within $30 \mathrm{~min}$. Cells in early-stage apoptosis (Annexin V positive, viability dye-negative) and late-stage apoptotic and necrotic cells (Annexin V, viability dye-positive) were distinguished, and the proportions were measured. The data were processed using MultiCycle software.

Cell cycle status was determined by staining the cellular DNA with PI, which was directly proportional to the amount of DNA within the cell. For cycle detection, cells were treated with RNase after fixation overnight in $70 \%$ ethanol. Cells were stained with PI for $30 \mathrm{~min}$ at $4^{\circ} \mathrm{C}$, and single cell suspensions of the samples were tested by FCM (FACSAria) passing through a $488 \mathrm{~nm}$ laser for excitation and fluorescence emissions were collected at $620 \mathrm{~nm}$ for PI. Cell cycle results were analyzed using the MultiCycle software.

Enzyme-linked immunosorbent assay (ELISA). Cells of 3 groups were cultured in complete medium (with 10\% FBS) under the same condition. Medium was refreshed when the cells reached $70 \%$ confluence. Cells were then washed by PBS and L-15 medium without FBS. The cells were incubated with serum-free medium for $20 \mathrm{~h}$. Concentrations of MMP9, MMP2, and VEGF in the cell culture supernatants of SW480 cells were quantified using MMP9, MMP2 and VEGF ELISA kits (R\&D Systems, Inc., USA). Each sample was analyzed in triplicate and manipulated according to the manufacturer's protocol.

Western blotting. Stably transfected cells of 3 groups were lysed in RIPA buffer [50 mM Tris (pH 7.4), $150 \mathrm{mM} \mathrm{NaCl}$,
$1 \%$ Triton $\mathrm{X}-100,0.1 \%$ SDS, $1 \%$ sodium deoxycholate, $5 \mathrm{mM}$ EDTA, $100 \mathrm{mM} \mathrm{NaF}$ and $1 \mathrm{mM} \mathrm{Na}_{3} \mathrm{VO}_{4}$ ] containing a protease inhibitor cocktail for $30 \mathrm{~min}$ on ice, followed by centrifuged for $30 \mathrm{~min}$ at 13,200 rpm. Protein concentrations were determined by the BCA method (Pierce, USA). Equal total proteins were subjected to electrophoresis by $12 \%$ SDS-PAGE gel, followed by transferring onto a PVDF membrane using a wet transblot system (Bio-Rad, Hercules, CA, USA). The membranes were blocked for $1 \mathrm{~h}$ at room temperature with $5 \%$ nonfat dry milk and incubated overnight at $4{ }^{\circ} \mathrm{C}$ with antibodies against DcR3, MT1-MMP, VEGF-C, VEGF-D and GAPDH (1:1,000). After washing, the membrane was incubated for $1 \mathrm{~h}$ with HRP-conjugated goat anti-rabbit secondary antibody diluted 1:5,000 in PBST. After further washing, the membrane was processed using SuperSignal West Pico Chemiluminescent Substrate (Pierce). The membrane was exposed to FujiFilm LAS3000 Image Reader (Fuji, Japan). The band densities of the western blots were normalized relative to the relevant GAPDH band density with ImageJ software (NIH).

Statistical analysis. All experiments were performed three times and the results were expressed as mean \pm SD. Statistical analysis was performed by SPSS 11.0. t-tests were used to compare the average values between two data populations. A P-value of less than 0.05 was considered to be statistically significant.

\section{Results}

Establishing stably transfected DcR3 RNAi SW480 cells. The expression level of DcR3 was decreased significantly in DcR3 RNAi SW480 cells compared to control cells $(\mathrm{P}<0.01)$ (Fig. 1C and D). This was also accompanied by high expression of GFP (Fig. 1B). Stable knockdown of DcR3 was still achieved after passaging the cells at least 10 times. 

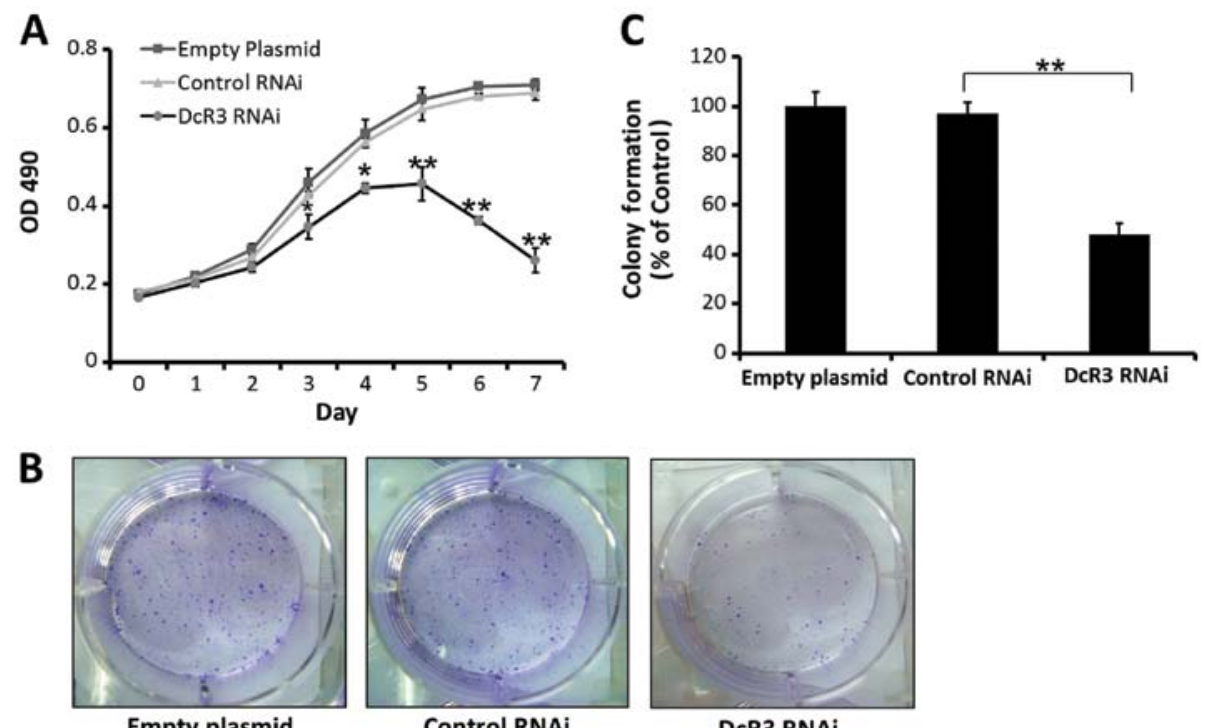

Control RNAi

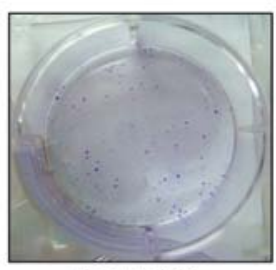

DCR3 RNAi

Figure 2. Effect of inhibiting DcR3 on growth and colony formation of SW480 cells. (A) Cell growth ability and viability of 3 groups (empty plasmid, control RNAi and DcR3 RNAi cells) by MTT assay. (B) Colony formation ability. (C) Quantitation from three independent assays. ${ }^{* *} \mathrm{P}<0.01,{ }^{*} \mathrm{P}<0.05$.

A
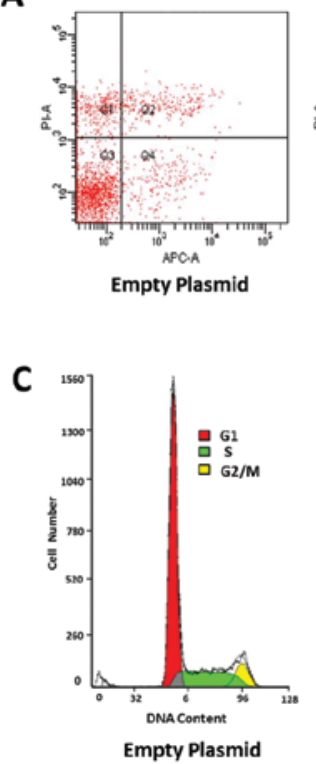

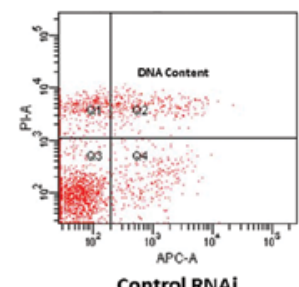

Control RNAI

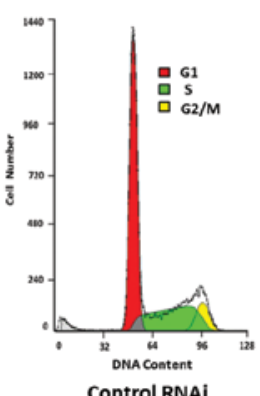

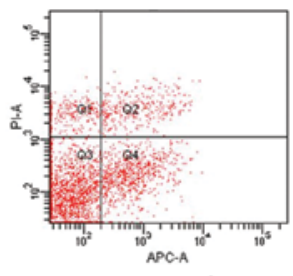

DCR3 RNAI

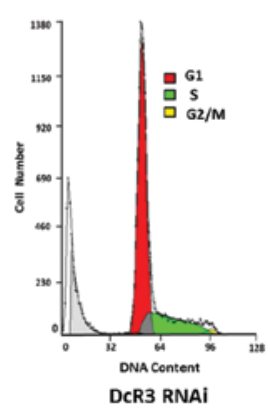

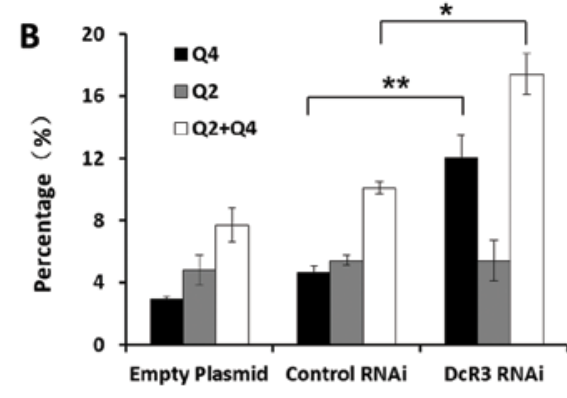

D

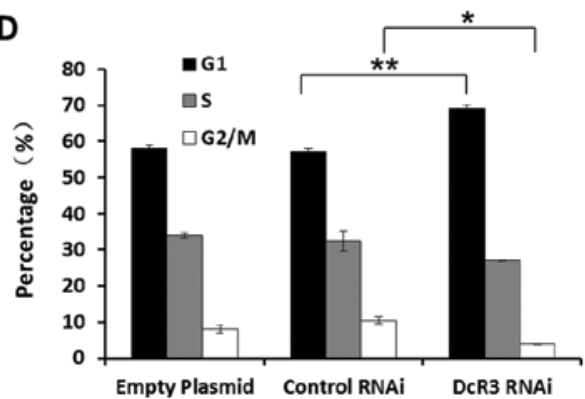

Figure 3. Effect of DcR3 knockdown on cell apoptosis and cell cycle of SW480 cells. (A and B) PI staining of cells transfected with empty plasmid, control RNAi and DcR3 RNAi. Cells with Annexin-V/PI double stain were tested by FCM. Q2, late-stage apoptotic and necrotic cells; Q4, late-stage apoptotic cells. $(\mathrm{C}$ and $\mathrm{D})$ Cells in each group were tested by FCM for cell cycle detection with PI staining. Shown are the proportion of each phase in cell cycle and the sub-G1 peak (apoptosis cells). ${ }^{* *} \mathrm{P}<0.01,{ }^{*} \mathrm{P}<0.05$.

Effects of decreasing DcR3 on the proliferation and colony formation of SW480 cells. A MTT growth assay showed that inhibiting DcR3 expression can restrain the growth of SW480 cells compared to control cells, according to the growth curves of 3 groups (Fig. 2A). Inhibiting DcR3 expression also significantly decrease the number of colony formation compared to control groups ( Fig. 2B and C).

Effects of inhibiting DcR3 on the apoptosis and cell cycle distribution of SW480 cells. Inhibiting DcR3 by RNAi induced apoptosis and G1 cell cycle arrest of SW480 cells compared to control cells (Fig. 3). The percentage of early- stage apoptotic cells was $12.0 \pm 1.5 \%$, which was higher than cells with empty plasmids $(2.9 \pm 0.2 \%)$ or with control RNAi plasmids $(4.7 \pm 0.4 \%)(\mathrm{P}<0.01)$. No observable effects were found on late-stage apoptotic and necrotic cells $(\mathrm{P}>0.05)$ (Fig. 3A and B). The cell cycle analysis showed a possible G1 phase arrest. The proportion of G1 phase cells increased, while decreased G2/M phase cells and unchanged S phase cells were observed. We also found that DcR3 RNAi cells contained a sub-G1 peak (cell debris) (Fig. 3C). Taken together, these results demonstrate that loss of DcR3 induced apoptosis and altered the cell cycle distribution of SW480 cells. 

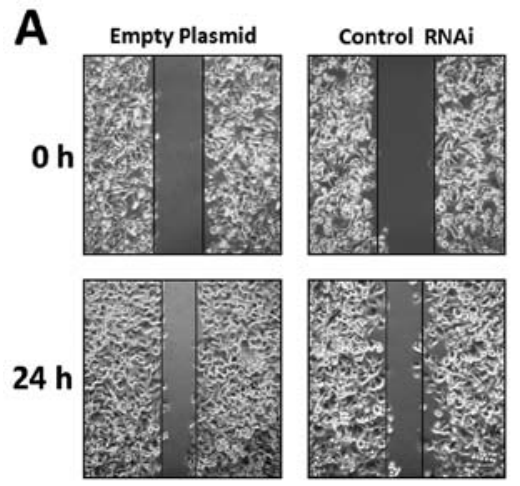

$48 \mathrm{~h}$
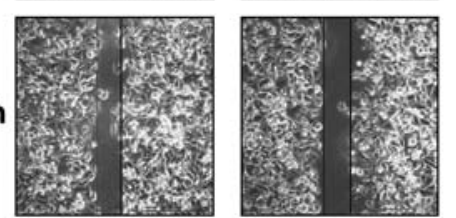

DCR3 RNAi
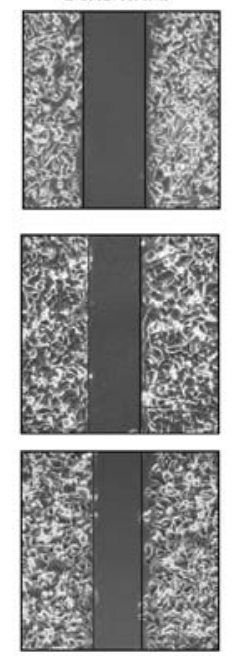

B

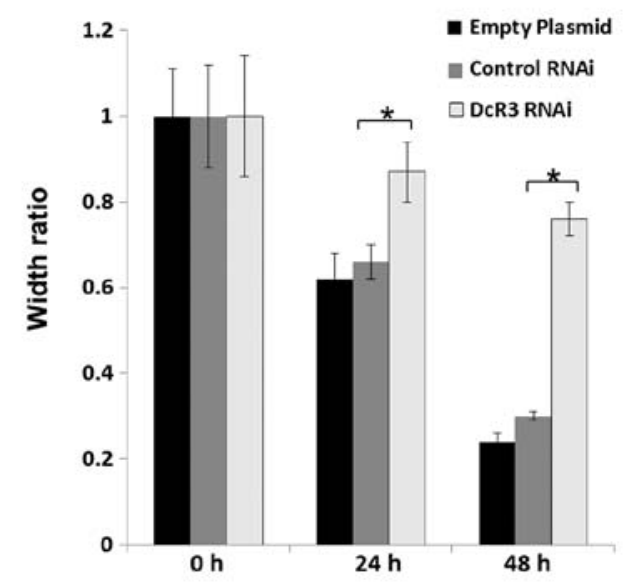

Figure 4. Effect of DcR3 knockdown on cell migration ability. (A) Cells were grown to $100 \%$ confluent and wounded by a sterile $100 \mu 1$ pipette tip. The ability of cell migration was monitored with an inverted microscope equipped with a digital camera. (B) Statistical analysis of three independent experiments. ${ }^{*} \mathrm{P}<0.05$.
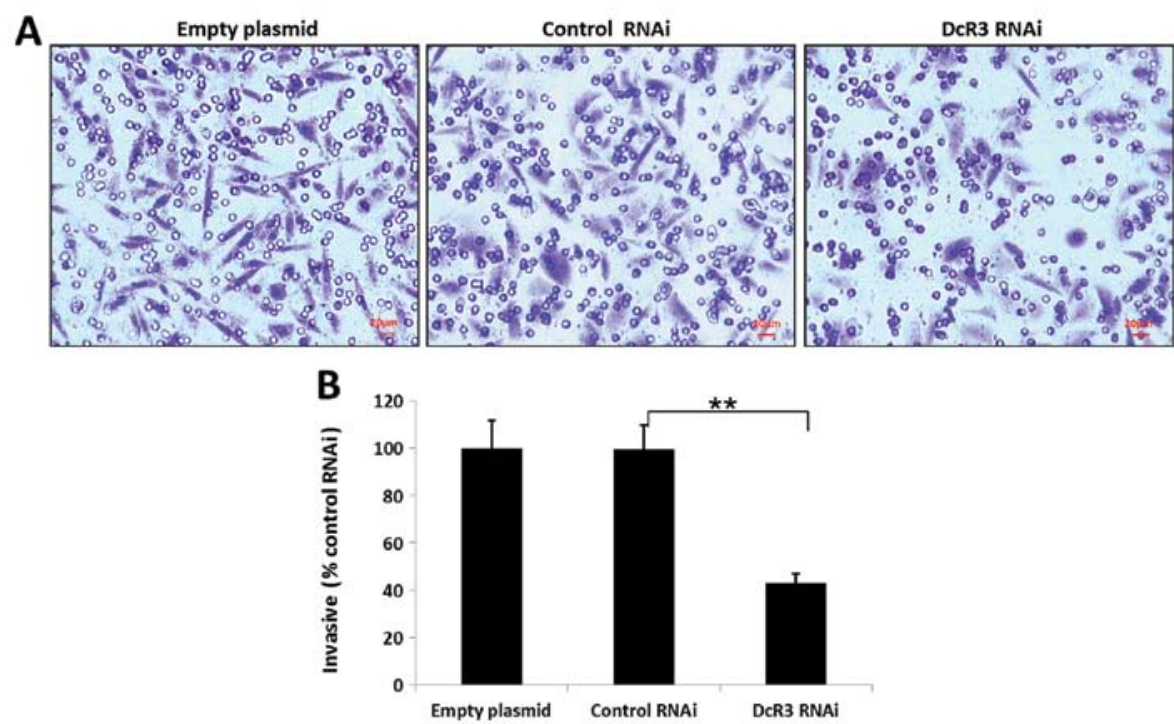

Figure 5. Effect of DcR3 knockdown on cell invasion of SW480 cells. (A) Cell invasion was assessed by a Transwell invasion assay. Cells were stained with crystal violet. (B) Twelve random fields of each test at $\mathrm{x} 400$ magnification were counted. ${ }^{* *} \mathrm{P}<0.01$.

Effects of inhibiting DcR3 on the migration and invasiveness of SW480 cells. To study whether DcR3 regulates the migration and invasion, we silenced its expression in SW480 cells and performed wound healing and Transwell assays. Knockdown of DcR3 was able to impair the migration (Fig. 4) and invasiveness (Fig. 5) of SW480 cells $(\mathrm{P}<0.01)$.

Effects of inhibiting DcR3 on the pro-metastatic gene expressions. Cancer cell metastasis is regulated by many proteins in vivo. To fully understand the molecular mechanism of DcR3 during metastasis, we determine the expression levels of some metastasis-associated proteins in cells of 3 groups. Extracellular levels of MMP2 (Fig. 6A), MMP9 (Fig. 6B), and VEGF (Fig. 6C) were significantly decreased compared to control cells. Loss of DcR3 in these cells also led to decrease endogenous expression of MT1-MMP, VEGF-C, VEGF-D (Fig. 7). These results indicated that the downregulation of DcR3 expression might inhibit the metastatic potential of SW480 cells by decreasing MT1-MMP, MMP2/9 and VEGF-C/D levels.

\section{Discussion}

Colon cancer is one of the most common malignancies in the world. In recent years, the morbidity and mortality of colon cancer has increased in China (9). Although significant improvement in diagnosing and treating colon cancer has been made in recent years, the advanced and metastatic disease remains a challenge. The 5-year survival rate after surgical resection in colon cancer patients with liver or lung metastases is approximately $30-50 \%(15,16)$. More importantly, no curative chemotherapeutic treatment is presently available for patients with metastatic disease. 

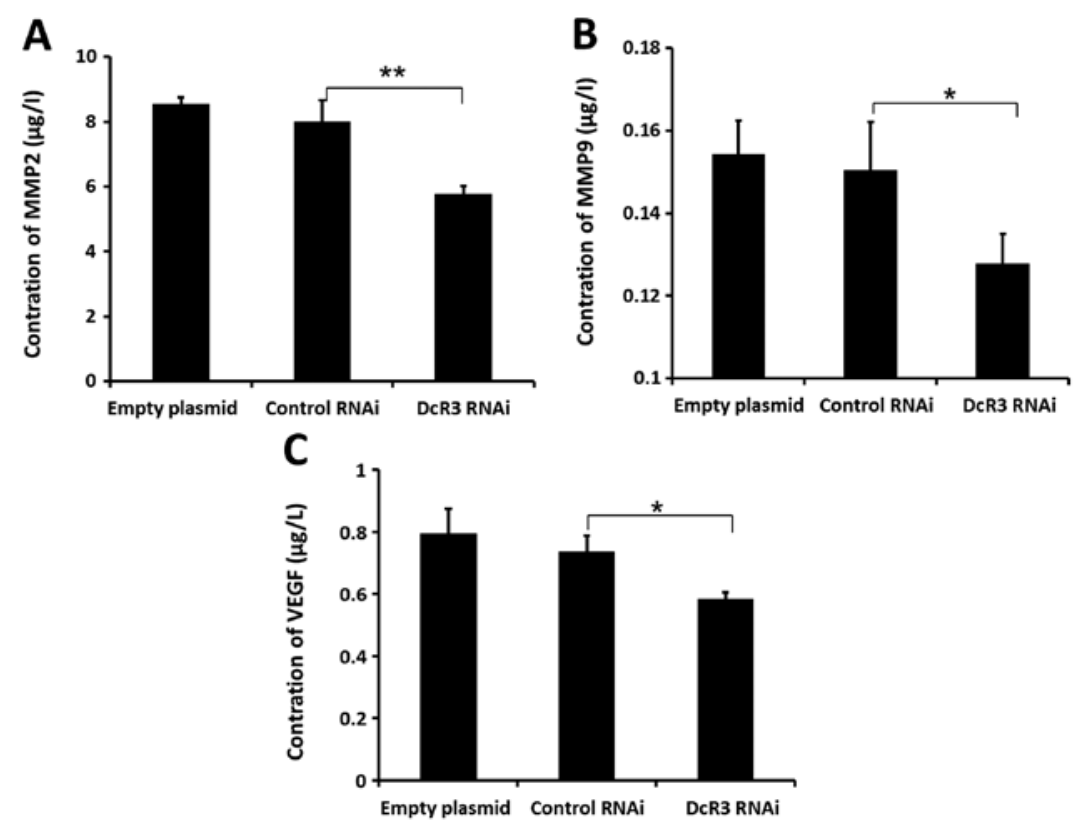

Figure 6. Effect of DcR3 RNAi on the expression of MMP2, MMP9 and VEGF. (A) The concentrations of MMP2 in cell culture supernatant of empty plasmid, control RNAi and DcR3 RNAi were detected by ELISA. (B) Levels of MMP9. (C) Levels of VEGF. Values shown are means \pm standard errors based on triplicate independent experiments. ${ }^{*} \mathrm{P}<0.05,{ }^{* *} \mathrm{P}<0.01$.
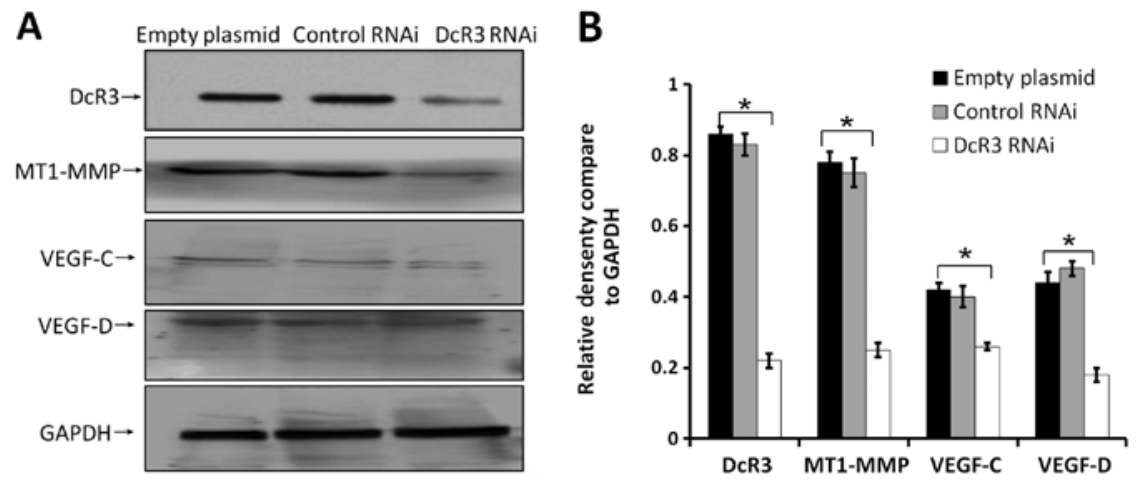

Figure 7. Effects of DcR3 RNAi on the expression of DcR3, MT1-MMP, VEGF-C and VEGF-D in SW480 cells. (A) Expression of DcR3, MT1-MMP, VEGF-C, VEGF-D detected by western blotting. (B) Protein levels were normalized to GAPDH. The data represent the mean \pm SE of relative density of bands from three independent experiments. ${ }^{*} \mathrm{P}<0.05$.

DcR3 protein belongs to the tumor necrosis factor family. Its expression is upregulated in several tumors, and its function during tumorigenesis has attracted extensive attention. Several studies have demonstrated that DcR3 can regulate cell proliferation, apoptosis and immune response by antagonizing FasL-, LIGH- and TLIA-mediated signals (17-19). DcR3 is closely related to tumor metastasis. However, the clinical significance in tumor metastasis of increased DcR3 levels have been confined to studying its relationship to clinical data $(6,8)$, and the molecular mechanism of DcR3 on tumor metastasis is rarely reported. In this study, we inhibited DcR3 expression by RNAi in SW480 cells with high original expression of the DcR3 gene (20). Loss of DcR3 inhibited the proliferation and induced apoptosis of these cells. We further demonstrated that DcR3 correlated to the growth of colon cancer cells. Besides, we investigated the change of metastatic ability and the underlying mechanism of DcR3 on SW480 cells.
As a greatly harmful disease to human health, the biological processes of colon cancer include tumorigenesis, progression, invasion and metastasis. The common tumor metastasis pathways include lymphatic, hematogenous and transcoelomic metastasises. Lymphatic metastasis is the behavior observed in the early stage of progression and metastasis of colon cancer. Patients with colon cancer often succumb to the disease due to multiple organ failure caused by blood metastasis in its advanced-stage. Pro-metastatic genes, changes in adhesion molecules, abnormal angiogenesis, matrix degradation and evasion in immune surveillance all contribute to the metastatic behavior of cancer cells. Angiogenesis, basement membrane (BM) and extracellular matrix (ECM) degradation are two essential steps during metastasis. Currently, the capability of angiogenesis is considered as an important character of malignancy $(21,22)$. Angiogenic regulators promote the growth of vascular endothelial cells and this is achieved in 
two ways: autocrine approach by vascular endothelial cells and paracrine pathway by tumor cells. In the process of tumor angiogenesis, the latter plays a main role. VEGF is a potent inducer of a variety of biological effects which was separated and purified firstly by Leung et al in 1989 (23). VEGF family members regulate normal physiological and pathological blood vessel growth. In recent years, studies have shown that macrophages and mast cells which invade into tumor tissue and tumor cells can secrete high levels of VEGF to stimulate the proliferation of tumor vascular endothelial cells through paracrine pathway. VEGF also increases vascular permeability, being beneficial to the recruitment of monocytes and fibroblasts, formation of tumor stroma and tumor cells getting into blood vessels, to enhance the metastatic potential of cancer cells $(24,25)$. Studies have shown that the expression of VEGF-C and VEGF-D was positive in 96 and $90 \%$ of colon and rectal cancers, respectively (26). VEGF-C and VEGF-D can stimulate the proliferation of lymphatic endothelial cells, resulting in lymphangiogenesis. VEGF-C and VEGF-D can also enhance lymphatic metastasis which has been identified as a prognostic indicator of certain types of tumors. However, different studies by Yang et al (27) have reported that the pro-angiogenic role of DcR3 does not depend on VEGFs. In our study, the silencing of DcR3 gene was associated with reduced levels of VEGF, VEGF-C and VEGF-D. The invasiveness and migration ability of SW480 cells were significantly impaired upon inhibiting DcR3. Knockdown of DcR3 also inhibited growth of xenograft tumors and decreased the microvessel and lymphatic vessel densities of tumor tissues. The mechanism by which DcR3 exerts its effects requires further studies.

Degradation of the extracellular matrix is an important stage during the metastatic spread of tumor cells to surrounding tissues. This is mediated by MMPs secreted by tumor cells. Therefore, MMPs have been associated with poor prognosis in a variety of cancers. The MMPs family includes 21 kinds of zinc metal ion-dependent proteolytic enzymes. Some studies have found that the expression levels of MMP2 and MMP9, which are the key enzymes in the degradation of $\mathrm{BM}$, were upregulated in a number of cancers (28). Recent studies have demonstrated that the MMPs can also directly regulate angiogenesis through factors such as MMP2, MMP9 and MMP14, in addition to ECM degradation (29,30). MMP2 is secreted by tumor and stromal cells through the zymogen form after hydrolysis (31), and able to degrade mucin IV and V, and collagen. Overexpression of DcR3 in human umbilical vein endothelial cells promoted cell proliferation, migration, MMP2 expression, tube formation and angiogenesis (27). In our studies, we also demonstrated a relationship between DcR3 and MMP2 expression. The activity of MMP2 was regulated by MT1-MMP, which is a type I transmembrane MMP. MT1-MMP activates MMP2 and MMP3 precursor as well as reconstruct the ECM (32). Additionally, MMP9 can also degrade ECM and BM located at the surface of tumor cells, allowing the invasion of these cells to surrounding tissue. Moreover, MMP9 plays an important role in angiogenesis and survival of metastatic tumor nodules. Some studies have indicated that MMPs, such as MMP9 and MT1-MMP, can regulate VEGFs expression. We show that inhibiting DcR3 also decreased MT1-MMP, MMP2 and MMP9 expres- sion, along with reduced migration and invasiveness of SW480 cells. We will further validate whether the downregulation of DcR3 can reduce enzymatic activity of MMPs.

Our results suggest that DcR3 regulates the growth and metastasis of colon cancer. DcR3, VEGFs and MMPS factors might play important roles during tumorigenesis and metastasis of colon cancer and interact with each other. However, how DcR3 regulates VEGFs and MMPs expression remains unclear. Our study suggests that DcR3 might serve as a therapeutic target for colon cancer.

\section{Acknowledgements}

The current study was supported by research grants from the National Natural Science Foundation of China (30972886).

\section{References}

1. Xiong G, Guo H, Ge X, Xu X, Yang X, Yang K, Jiang Y and Bai Y: Decoy receptor 3 expression in esophageal squamous cell carcinoma: correlation with tumour invasion and metastasis. Biomarkers 16: 155-160, 2011.

2. Colucci S, Brunetti G, Mori G, Oranger A, Centonze M, Mori C, Cantatore FP, Tamma R, Rizzi R, Liso V, Zallone A and Grano M: Soluble decoy receptor 3 modulates the survival and formation of osteoclasts from multiple myeloma bone disease patients. Leukemia 23: 2139-2146, 2009

3. Connor JP and Felder M: Ascites from epithelial ovarian cancer contain high levels of functional decoy receptor 3 (DcR3) and is associated with platinum resistance. Gynecol Oncol 111: 330-335, 2008.

4. Xiong G, Guo H, Wang K, Hu H, Wang D, Xu X, Guan X, Yang K and Bai Y: Polymorphisms of decoy receptor 3 are associated with risk of esophageal squamous cell carcinoma in Chinese Han. Tumour Biol 31: 443-449, 2010.

5. Chen J, Zhang L and Kim S: Quantification and detection of DcR3, a decoy receptor in TNFR family. J Immunol Methods 285: 63-70, 2004.

6. Wu Y, Han B, Sheng H, Lin M, Moore PA, Zhang J and Wu J: Clinical significance of detecting elevated serum DcR3/TR6/M68 in malignant tumor patients. Int J Cancer 105: 724-732, 2003.

7. Tsuji S, Hosotani R, Yonehara S, et al: Endogenous decoy receptor 3 blocks the growth inhibition signals mediated by Fas ligand in human pancreatic adenocarcinoma. Int J Cancer 106: 17-25, 2003.

8. Macher-Goeppinger S, Aulmann S, Wagener N, Funke B, Tagscherer KE, Haferkamp A, Hohenfellner M, Kim S, Autschbach F, Schirmacher P and Roth W: Decoy receptor 3 is a prognostic factor in renal cell cancer. Neoplasia 10: 1049-1056, 2008.

9. Jemal A, Murray T, Ward E, Samuels A, Tiwari RC, Ghafoor A, Feuer EJ and Thun MJ: Cancer statistics, 2005. CA Cancer J Clin 55: 10-30, 2005.

10. Lee WS, Kang, M, Baek JH, Lee JI and Ha SY: Clinical impact of tumor-infiltrating lymphocytes for survival in curatively resected stage IV colon cancer with isolated liver or lung metastasis. Ann Surg Oncol 20: 697-702, 2013.

11. Jagani H, Rao JV, Palanimuthu VR, Hariharapura RC and Gang SA: A nanoformulation of siRNA and its role in cancer therapy: in vitro and in vivo evaluation. Cell Mol Biol Lett 18: 120-136, 2013.

12. Okamoto S, Amaishi Y, Goto Y, Ikeda H, Fujiwara H, Kuzushima K, Yasukawa M, Shiku $\mathrm{H}$ and Mineno J: A promising vector for TCR gene therapy: differential effect of siRNA, 2A peptide, and disulfide bond on the introduced TCR expression. Mol Ther Nucleic Acids 1: e63, 2012.

13. Baker AM, Cox TR, Bird D, Lang G, Murray GI, Sun XF, Southall SM, Wilson JR and Erler JT: The role of lysyl oxidase in SRC-dependent proliferation and metastasis of colorectal cancer. J Natl Cancer Inst 103: 407-424, 2011.

14. Gulhati P, Cai Q, Li J, Liu J, Rychahou PG, Qiu S, Lee EY, Silva SR, Bowen KA, Gao T and Evers BM: Targeted inhibition of mammalian target of rapamycin signaling inhibits tumorigenesis of colorectal cancer. Clin Cancer Res 15: 7207-7216, 2009. 
15. Binkhathlan $\mathrm{Z}$ and Alshamsan A: Emerging nanodelivery strategies of RNAi molecules for colon cancer therapy: preclinical developments. Ther Deliv 3: 1117-1130, 2012.

16. Wu B, Zhou $\mathrm{H}, \mathrm{Hu} \mathrm{L}, \mathrm{Mu} \mathrm{Y}$ and $\mathrm{Wu} \mathrm{Y}$ : Involvement of PKCa activation in TF/VIIa/PAR2-induced proliferation, migration, and survival of colon cancer cell SW620. Tumour Biol 34: 837-846, 2013.

17. Yu KY, Kwon B, Ni J, Zhai Y, Ebner R and Kwon BS: A newly identified member of tumor necrosis factor receptor superfamily (TR6) suppresses LIGHT-mediated apoptosis. J Biol Chem 274 13733-13736, 1999.

18. Shi G, Mao J, Yu G, Zhang J and Wu J: Tumor vaccine based on cell surface expression of DcR3/TR6. J Immunol 174: 4727-4735, 2005.

19. Takahama Y, Yamada Y, Emoto K, Fujimoto H, Takayama T, Ueno M, Uchida H, Hirao S, Mizuno T and Nakajima Y: The prognostic significance of overexpression of the decoy receptor for Fas ligand (DcR3) in patients with gastric carcinomas. Gastric Cancer 5: 61-68, 2002.

20. Bai C, Connolly B, Metzker ML, Hilliard CA, Liu X, Sandig V, Soderman A, Galloway SM, Liu Q, Austin CP and Caskey CT: Overexpreasion of M68/DcR3 in human gastrointestinal tract tumors independent of gene amplificalion and its location in a four-gene cluster. Proc Natl Acad Sci USA 97: 1230-1235, 2000 .

21. Flokman $\mathbf{J}$ and D'amore PA: Blood vessel formation: what is its molecular basis? Cell 87: 1153-1155, 1996.

22. Risau W: Mechanisms of angiogenesis. Nature 386: 671-674, 1997.

23. Lepelletier Y, Camara-Clayette V, Jin H, Hermant A, Coulon S, Dussiot M, et al: Prevention of mantle lymphoma tumor by routing transferrin receptor lysosomal compartment. Cancer Res 67: 1145-1154, 2007.

24. Stacker SA, Caesar C, Baldwin ME, et al: VEGF-D promotes the metastatic spread of tumor cells via the lymphatics. Nat Med 7: 186-191, 2001.
25. Nagy JA, Vasile E, Feng D, et al: Vascular permeability factor/vascular endothelial growth factor induces lymphangiogenesis as well as angiogenesis. J Exp Med 196: 1497-1506, 2002.

26. George ML, Tutton MG, Janssen F, Arnaout A, Abulafi AM, Eccles SA and Swift RI: VEGF-A, VEGF-C, and VEGF-D in colorectal cancer progression. Neoplasia 3: 420-427, 2001.

27. Yang CR, Hsieh SL, Teng CM, Ho FM, Su WL and Lin WW: Soluble decoy receptor 3 induces angiogenesis by neutralization of TL1A, a cytokine belonging to tumor necrosis factor superfamily and exhibiting angiostatic action. Cancer Res 64: 1122-1129, 2004.

28. Egeblad M and Werb Z: New functions for the matrix metalloproteinases in cancer progression. Nat Rev Cancer 2: 161-174, 2002.

29. Gao J, Ding F, Liu Q and Yao Y: Knockdown of MACC1 expression suppressed hepatocellular carcinoma cell migration and invasion and inhibited expression of MMP2 and MMP9. Mol Cell Biochem 376: 21-32, 2013.

30. Hotary KB, Allen ED, Brooks PC, Datta NS, Long MW and Weiss SJ: Membrane type I matrix metalloproteinase usurps tumor growth control imposed by the three-dimensional extracellular matrix. Cell 114: 33-45, 2003

31. Yamashita K, Upadhay S, Mimori K, Inoue H and Mori M: Clinical significance of secreted protein acidic and rich in cystein in esophageal carcinoma and its relation to carcinoma progression. Cancer 97: 2412-2419, 2003.

32. Koyama H, Iwata H, Kuwabara Y, Iwase H, Kobayashi S and Fujii Y: Gelatinolytic activity of matrix metalloproteinase-2 and -9 in oesophageal carcinoma; a study using in situ zymography. Eur J Cancer 36: 2164-2170, 2000. 